\title{
MRI-derived neurovascular compression score provides the indication of surgical treatment of patients with primary trigeminal neuralgia: $A$ validated multi-center study
}

Jian Shen

Huzhou Central Hospital

Wenli Zhou

Zhejiang University School of Medicine

Weiqiang Shen

Huzhou Central Hospital

Huimei Zhang

Huzhou Central Hospital

Xianfang Hu

Huzhou Central Hospital

Wei Lin

Zhejiang University School of Medicine

Xiaoli Gu

Shanghai Guanghua Hospital of Intergrated Traditional Medicine and Western Medicine

Hongjie Hu ( $\nabla$ hongjiehu@zju.edu.cn )

Zhejiang University School of Medicine Sir Run Run Shaw Hospital https://orcid.org/0000-0003-29858601

Research article

Keywords: trigeminal neuralgia, MRI, neurovascular compression, 3D-TOF, FIESTA

Posted Date: July 8th, 2020

DOI: https://doi.org/10.21203/rs.3.rs-40269/v1

License: (c) (i) This work is licensed under a Creative Commons Attribution 4.0 International License.

Read Full License 


\section{Abstract \\ Background}

The radiological assessment of neurovascular compression (NVC) is various regarding MRI techniques and assessing methods in patients with trigeminal neuralgia (TN), and the false-positive rate of MRI findings is not low. Better MRI techniques with the NVC assessing method are warranted to be determined. This study aims to investigate the diagnostic performance of 3D TOF MRA and 3D Fast Imaging Employing Steady-state Acquisition (FIESTA) with a novel NVC scoring system in TN patients.

\section{Methods}

Patients with confirmed TN who underwent MRI studies before microvascular decompression (MVD) were retrospectively included into the study. A new NVC scoring system based on the contact relationship of the trigeminal nerve and the vessel was performed to assess the NVC in the symptomatic and contralateral asymptomatic side. The radiological finding was correlated with the intraoperative result to figure out the diagnostic accuracy of MRI techniques. Besides, the comparison of both sides was performed to determine the radiological indicator of MVD.

\section{Results}

Seventy-three TN patients were recruited, and 146 trigeminal nerve sides were analyzed. For the symptomatic sides, 69 patients had surgically confirmed offending vessels, most of which was SCA, and the positive NVC rate was $95.5 \%$. For the contralateral side, 33 patients have been found with NVC on MRI. The NVC score of the symptomatic side was significantly higher than that of asymptomatic sides (6.7 vs. 1.6; $p<0.001$ ). The optimal cut-off value in predicting trigeminal neuralgia was found as NVC > 4 with sensitivity and specificity of $82.2 \%$ and $98.6 \%$, respectively.

\section{Conclusion}

3D-TOF MRA and FIESTA enable a good diagnostic performance of NVC, and NVC score $>4$ was identified to predict trigeminal neuralgia, suggestive of subsequent surgical treatment.

\section{Trial registration:}

The study has been retrospectively registered at the local ethical Institution Review Board (IRB) of Huzhou Central Hospital and Sir Run Run Shaw Hospital with the IRB number (20181108-01; Huzhou) and (20200423-43; SRRSH). 


\section{Introduction}

Trigeminal neuralgia (TN) is an exemplary condition of neuropathic facial pain, characterized as shortlasting episodes of unilateral electric shock-like pain with abrupt onset and termination(1,2). Patients usually had characteristic paroxysmal pain and concomitant continuous pain in $50 \%$ of patients(2). It has affected 4.3-27 per 100,000 people per year worldwide with high prevalence among women and increased with age (3). In terms of pathophysiology, TN can be divided into two types-classical TN or secondary TN caused by other neurological diseases, such as multiple sclerosis and cerebral tumors(3). The classical TN more frequently affects the left side and the 2nd and/or 3rd trigeminal division(4).

Approximately $30 \%-70 \%$ of TN patients were due to the compressed trigeminal nerve caused by surrounding vessels in the cerebellopontine cistern, which is well-known as neurovascular compression (NVC) (5-8). Sodium channel blockers are perceived as the first-line treatment, effective in most TN patients, and surgical treatment is commonly administered in patients with TN despite given adequate medication in relieving symptoms, such as microvascular decompression (MVD). Neuroimaging plays an essential role in the TN diagnosis and preoperative evaluation of the existence and severity of NVC, especially in the early workup(9).

There are various magnetic resonance imaging (MRI) techniques in assessing NVC, including highresolution three-dimensional (3D) time-of-flight (TOF) MR angiography (MRA), gadolinium (Gad)enhanced 3D spoiled gradient-recalled imaging, 3D T2-weighted imaging(8). To date, there was no consensus on MR techniques and the evaluation method of NVC. Previous studies have reported the sensitivity and specificity of imaging sequences in detecting NVC varied, respectively, from $67-100 \%$ and from $50-100 \%(8,9)$. NVC can occur in TN patients without symptom, whereas TN can also occur in the absence of $\mathrm{NVC}(10)$.

Taken together, current evidence implies that there is a discrepancy between the radiological and surgical NVC assessment of patients with suspected TN. The predictive accuracy of MRI needs to be improved to better guide the surgical decision by applying more proper MRI techniques and assessing methods. Therefore, this study would investigate the predictive value of MRI techniques-3D TOF MRA combined with 3D Fast Imaging Employing Steady-state Acquisition (FIESTA) by using a new NVC scoring system. This study also aims to determine the radiological indicators of MVD to improve TN patients' clinical outcomes.

\section{Methods}

\section{Patient cohort and study design}

Patients with single-site TN and underwent MVD at Huzhou Central Hospital and Sir Run Run Shaw Hospital (SRRSH) from January 2011 to December 2015 were retrospectively included into the study. The TN was assessed and diagnosed by specialized neurologists based on the International Classification of Headache Disorders(11). All patients did MRI scans within one week before the MVD surgery, and 
symptomatic patients caused by other neurological diseases, such as brain tumors, multiple sclerosis, were excluded from the study.

All patients were assessed for NVC status on MRI imaging, including both symptomatic and asymptomatic sides. A radiological NVC scoring system was performed to assess the NVC quantitatively(12), linking to the surgical findings. The study has been approved by local ethical Institution Review Board (IRB) of Huzhou Central Hospital and Sir Run Run Shaw Hospital with the IRB number (20181108-01; Huzhou) and (20200423-43; SRRSH). The patient consent form was waived.

\section{Imaging Protocol}

Patients at Huzhou Central Hospital underwent a preoperative MRI exam by using a 3.0-T MR scanner(GE Discovery 750), and patients at Sir Run Run Shaw Hospital were scanned by 1.5-T GE Signa Excite and GE 3.0-T Signa HD. 3D-FIESTA and 3D-TOF were acquired with the following parameters: time of repeat (TR) $5.0 \mathrm{~ms}$, time of echo (TE) $2.3 \mathrm{~ms}$, matrix size $256 \times 256$, slice thickness $1.0 \mathrm{~mm}$, and TR $11.0 \mathrm{~ms}$, TR $2.6 \mathrm{~ms}$, matrix size $384 \times 240$, slice thickness $1.0 \mathrm{~mm}$, respectively. The scan baseline was parallel to the auditory basal line, extending from the bottom of the frontal to the pontomedullary sulcus. The trigeminal nerve, facial auditory nerve and main branches of the vertebral artery were then included.

\section{Imaging post-processing}

The cross-sectional image was analyzed at GE advanced workstation (version 4.6). 3D-FIESTA and 3DTOF MRA sequences were reconstructed to get the coronal and oblique sagittal images by performing the Reformat module with a slice thickness of $1.0 \mathrm{~mm}$. The relationship between the vessel and the trigeminal nerve was then evaluated in three different planes. 3D MIP was used to reconstruct TOF images to assess the course and origin of the target vessel.

\section{Radiologic assessment of NVC}

Two neuroradiologists were blinded to assess MR exams. A NVC scoring system was adapted from Chen et al's study(12) to assess the relationship of the vessel and trigeminal nerve. The system had four degrees. Score 0 referred no relationship between the nerve and the vessel, or the relationship is difficult to be evaluated. If the vessel crossed or touched the nerve but without any root deformity and cerebrospinal fluid, this case was graded as 1 . If there was a significant indentation on the trigeminal nerve root caused by the compression of the offending vessel, the score increased to 2 . The severe degree was score 3, which was given by an existence of a distortion and/or a displacement of the compressed root, compared with the asymptomatic side. Besides, the overall NVC score was calculated in the sum of three scores assessed separately from axial, oblique sagittal, and coronal images. The assessment illustration was seen in Figure 1.

\section{Surgical procedure}


Patients who were suitable for general anesthesia and posterior cranial fossa exploration were proceeded with MVD. A retrosigmoid incision was performed in all patients. The arachnoid was cleared to expose the cerebellopontine angle and its containing structures. After opening the cistern and then draining the cerebrospinal fluid, the trigeminal nerve was exposed. The surrounding arachnoid was cleared under the endoscope to visualize the offending vessels, which were separated from the nerve with a folded Teflon patch afterward. The nerves without any NVC were confirmed before closing the dura mater. The drainage tube was inserted if necessary.

\section{Statistical analysis}

The data was presented as mean \pm standard deviation or numbers with the percentage in the blanket. The NVC score comparison between symptomatic and asymptomatic sides was assessed by the independent $t$-test. The inter and intra-observer variability assessment of the NVC score was calculated by the intraclass correlation coefficient (ICC) based on 40 cases, which were randomly selected. The receiver operating characteristic (ROC) curve was applied to determine the optimal cut-off value in predicting trifacial neuralgia. A $p<0.05$ was regarded as statistically significant for all statistical tests. All the statistical analyses were performed using SPSS Statistics software (version 23), and ROC curves were drawn by MedCalc 19.1.2.

\section{Results}

\section{Patient cohort}

Seventy-three patients were included into the study, and $32.9 \%(24 / 73)$ were males. The mean age of this cohort was $60 \pm 9$ years, ranging from 38 to 77 years old. The side of the symptom was approximately 1:1 for left versus right. The symptom of patients was predominately distributed along with V2 with the prevalence of $49.3 \%$, compared to other branches. And twenty-two (30.1\%) patients had more than one branch involved. The symptom duration of this cohort was four years on average (range: 0.3-20 years). For preoperative treatment, carbamazepine was predominantly administrated. The overall information on clinical characteristics was summarized in Table 1.

\section{Surgical findings and its correlation with MRI}

All patients were proceeded with MVD surgery, and both symptomatic and asymptomatic sides were studies for NVC assessment. For the symptomatic side, 69 patients had offending vessels, and the NVC positive rate confirmed by surgery was $94.5 \%$ (69/73). Superior cerebellar artery (SCA) was the most involved offending vessel (49.3\%). The false-positive findings in MRI among four patients were due to venule in three cases and arachnoid adhesion in one case. For the asymptomatic side, NVC was observed in MRI in thirty-three patients. SCA were also the predominant offending vessels in the asymptomatic side. The overall surgical findings were shown in Table 2.

\section{NVC score assessment}


Sixty-four cases were performed at Sir Run Run Shaw Hospital (1.5T: 45 cases and 3.0T: 19 cases). Nine patients were recruited from Huzhou Central Hospital. The intra-observer and inter-observer variability of NVC measurement for the symptomatic side showed strong agreement with ICC of $0.95(p<0.001)$ and ICC of 0.90 ( $p<0.001)$, respectively. For the asymptomatic side, the ICC of the intra-observer and interobserver variability was $0.92(p<0.001)$ and $0.94(p<0.001)$. The NVC score of the symptomatic side was $6.7 \pm 2.2$ on average.

Interestingly, there were thirty-three patients having NVC at both sides but with a single-side pain. For the asymptomatic side, the average NVC score was significantly lower than that of the symptomatic side (1.6 vs. 6.7; p 0.001). A total of 146 sides with NVC were performed with ROC analysis. The result showed that the optimal cut-off value in predicting trifacial neuralgia was NVC $>4$ with sensitivity and specificity of $82.2 \%$ and $98.6 \%$, respectively (AUC=0.97; $p<0.001)$, which suggested a radiological indicator of MVD surgery. The ROC curve and analyzed data were seen in Figure 2 and Table 3.

\section{Discussion}

This study applied a radiological scoring system assessing the relationship of the trigeminal nerve and the vessel based on MRI images in 73 patients with single-side TN. NVC severity was evaluated on both symptomatic and asymptomatic sides in a total of 146, correlating the results with surgical findings. For the symptomatic sides, 69 patients had surgically confirmed offending vessels, most of which was SCA, and the positive NVC rate was $95.5 \%$. The NVC score of the symptomatic side was significantly higher than that of asymptomatic sides (6.7 vs. 1.6; $p<0.001)$. The optimal cut-off value in predicting TN was found as NVC > 4 with sensitivity and specificity of $82.2 \%$ and $98.6 \%$, respectively, which provided a radiological indicator in MVD non-invasively, guiding the neurologists with the decision- making.

MVD is the primary option and carry a low risk of requiring a secondary procedure and facial numbness after surgery $(13,14)$. A recent prospective study investigating the outcomes of MVD in patients with TN has found that male gender and NVC with morphological changes on preoperative MRI images, where distortion, dislocation, and distension were observed, predicted the excellent outcomes(15). This study and earlier studies have reported that the severity degree of NVC was significantly associated with surgical outcomes $(16,17)$. The underlying mechanism may due to the fact that the demyelinated trigeminal nerve root remyelinates after MVD and the neuroedema at the symptomatic NVC were released $(3,18)$. Therefore, the preoperative neurological imaging assessment of NVC severity is important in predicting patients' outcomes beyond diagnosis $(15,19)$.

Previous studies have shown the various methods in assessing NVC and its severity by different MRI sequences. The present study using 3D-TOF MRA and FIESTA has achieved high diagnostic accuracy as 94.5\% for symptomatic nerve. For the assessment method, some studies only performed dichotomous assessment methods such as patients with or without $\operatorname{NVC}(20,21)$. T. Satoh et al. classified the NVC severity into four grades according to the extent of the nerve circumference in contact with the vessel(22). NVC with the vessel contacting the trigeminal nerve covering $>20 \%$ was defined as severe and that with $<$ 
$20 \%$ was moderate. Another two grades were simple NVC with slight touch and no contact. This method only assessing the compressed extent may underestimate the severe situation with morphological changes, which was critical in clinical diagnosis and prognosis(9).

More recent studies have further developed the NVC evaluation system, particularly focusing on the morphological changes of the trigeminal nerve. Tone Bruvik Heinskou's group has graded the NVC contact as simple contact, displacement (displacement or distortion), and atrophy with a reduced volume of the nerve(15). The present study also included the morphological changes and adapted a scoring system in describing the NVC degree in a quantitative manner but in more detail (12). The assessment taking the average value of three directions - axial, oblique sagittal, and coronal can improve the diagnostic accuracy of NVC. Besides, to the best of our knowledge, this study was the first stud to determine a cut-off value as a radiological indicator of MVD with NVC score $>4$, which was more practical in real clinical settings.

NVC without morphological changes-a simple contact commonly occurred in patients with facial pain or even without causing symptoms $(6,23,24)$, which was considered as a normal neuroanatomic variant of patients with facial pain but not fulfilling the trifacial neuralgia diagnostic criteria(3,9). And these patients had the tendency to develop into classical paroxysmal pain, which indicated the importance of follow-up for these patients. An earlier study investigating the radiographic performance of NVC in patients with and without TN has also found that the compression of the proximal nerve and nerve indentation or displacement were significant predictors of TN(6). The present study with the result of NVC $>4$ showed a good agreement in predicting TN. This study has identified a prevalence of $33 \%$ of NVC occurring in asymptomatic nerve with score mostly around 1-3, suggesting patients with NVC approaching four needs more clinical attention and more intensive imaging follow-up.

\section{Conclusion}

3D-TOF MRA and FIESTA enable an excellent diagnostic performance of NVC, and NVC score $>4$ was identified to predict TN, suggestive of subsequent surgical treatment.

\section{Abbreviations}

AICA Anterior inferior cerebellar artery

FIESTA Fast Imaging Employing Steady-state Acquisition

ICC Intraclass correlation coefficient

MRA MR angiography

MRI Magnetic resonance imaging

MVD Microvascular decompression 
NVC Neurovascular compression

PICA Posterior inferior cerebellar artery

ROC Receiver operating characteristic

SCA Superior cerebellar artery

SRRSH Sir Run Run Shaw Hospital

TN Trigeminal neuralgia

TOF Time-of-flight

\section{Declarations}

\section{Ethics approval and consent to participate}

The study has been approved by the local ethical Institution Review Board (IRB) of Huzhou Central Hospital and Sir Run Run Shaw Hospital with the IRB number (20181108-01; Huzhou) and (20200423-43; SRRSH). The patient consent form was waived.

\section{Consent for publication}

As stated in the ethics approval section, the patient consent form was waived which was granted that it was a retrospective imaging study carrying no risk in patients. The waiver request was approved by the local ethical Institution Review Board (IRB) of Huzhou Central Hospital and Sir Run Run Shaw Hospital.

\section{Availability of data and materials}

Not applicable.

\section{Competing interests}

The authors declare that they have no competing interests.

\section{Funding}

This study is supported by funding from Science and Technology Bureau Program of Huzhou (No. 2018GYB56).

\section{Authors' contributions}


Jian Shen and Wenli Zhou designed the study and wrote the manuscript. Jianshen, Weiqiang Shen, Xianfang Hu, Huimei Zhang and Wei Lin assessed the MRI images and performed the post-processing work. Wenli Zhou analyzed and interpreted the patient data regarding NVC performance. Jian Shen and Wenli Zhou contributed equally to the work acting as co-first authors. Xiaoli Gu and Hongjie Hu were cocorresponding authors supervised and helped with the whole project. All authors read and approved the final manuscript.

\section{Acknowledgements}

Not applicable.

\section{References}

1. Bašić Kes V, Zadro Matovina L (2017) Accommodation to Diagnosis of Trigeminal Neuralgia. Acta Clin Croat 56(1):157-161

2. Cruccu G. Trigeminal Neuralgia. Continuum (Minneap Minn). 2017;23(2, Selected Topics in Outpatient Neurology):396-420

3. Maarbjerg S, Di Stefano G, Bendtsen L, Cruccu G (2017) Trigeminal neuralgia - diagnosis and treatment. Cephalalgia 37(7):648-657

4. Maarbjerg S, Gozalov A, Olesen J, Bendtsen L (2014) Trigeminal Neuralgia - A Prospective Systematic Study of Clinical Characteristics in 158 Patients. Headache: The Journal of Head Face Pain 54(10):1574-1582

5. Dandy WE (1934) Concerning the cause of trigeminal neuralgia. The American Journal of Surgery 24(2):447-455

6. Miller JP, Acar F, Hamilton BE, Burchiel KJ (2009) Radiographic evaluation of trigeminal neurovascular compression in patients with and without trigeminal neuralgia. J Neurosurg 110(4):627-632

7. Magown P, Ko AL, Burchiel KJ (2019) The Spectrum of Trigeminal Neuralgia Without Neurovascular Compression. Neurosurgery 85(3):E553-E559

8. Anderson VC, Berryhill PC, Sandquist MA, Ciaverella DP, Nesbit GM, Burchiel KJ (2006) Highresolution three-dimensional magnetic resonance angiography and three-dimensional spoiled gradient-recalled imaging in the evaluation of neurovascular compression in patients with trigeminal neuralgia: a double-blind pilot study. Neurosurgery 58(4):666-673; discussion - 73

9. Bendtsen L, Zakrzewska JM, Abbott J, Braschinsky M, Di Stefano G, Donnet A et al (2019) European Academy of Neurology guideline on trigeminal neuralgia. Eur J Neurol 26(6):831-849

10. Lee A, McCartney S, Burbidge C, Raslan AM, Burchiel KJ (2014) Trigeminal neuralgia occurs and recurs in the absence of neurovascular compression. J Neurosurg 120(5):1048-1054 
11. The International Classification of Headache Disorders, 3rd edition (beta version). Cephalalgia. 2013;33(9):629-808

12. Chen MJ, Zhang WJ, Guo ZL, Yang C, Zhang WH, Dong MJ et al (2014) Preoperative evaluation of the neurovascular compression using magnetic resonance tomographic angiography: our radiologic indications for microvascular decompression to treat trigeminal neuralgia. J Craniofac Surg 25(4):e384-e388

13. Li Y, Yang L, Ni J, Dou Z (2019) Microvascular decompression and radiofrequency for the treatment of trigeminal neuralgia: a meta-analysis. J Pain Res 12:1937-1945

14. Cruccu G, Gronseth G, Alksne J, Argoff C, Brainin M, Burchiel K et al (2008) AAN-EFNS guidelines on trigeminal neuralgia management. Eur J Neurol 15(10):1013-1028

15. Heinskou TB, Rochat P, Maarbjerg S, Wolfram F, Brennum J, Olesen J et al (2019) Prognostic factors for outcome of microvascular decompression in trigeminal neuralgia: A prospective systematic study using independent assessors. Cephalalgia 39(2):197-208

16. Barker FG, Jannetta PJ, Bissonette DJ, Larkins MV, Jho HD (1996) The Long-Term Outcome of Microvascular Decompression for Trigeminal Neuralgia. N Engl J Med 334(17):1077-1084

17. Sindou M, Leston J, Decullier E, Chapuis F (2007) Microvascular decompression for primary trigeminal neuralgia: long-term effectiveness and prognostic factors in a series of 362 consecutive patients with clear-cut neurovascular conflicts who underwent pure decompression. J Neurosurg 107(6):1144-1153

18. DeSouza DD, Davis KD, Hodaie M (2015) Reversal of insular and microstructural nerve abnormalities following effective surgical treatment for trigeminal neuralgia. Pain 156(6):1112-1123

19. Duan Y, Sweet J, Munyon C, Miller J (2015) Degree of distal trigeminal nerve atrophy predicts outcome after microvascular decompression for Type 1a trigeminal neuralgia. J Neurosurg 123(6):1512-1518

20. Vergani F, Panaretos P, Penalosa A, English P, Nicholson C, Jenkins A (2011) Preoperative MRI/MRA for microvascular decompression in trigeminal neuralgia: consecutive series of 67 patients. Acta Neurochir 153(12):2377-2382

21. Zhou Q, Liu Z, Li C, Qu C, Ni S, Zeng Q (2011) Preoperative evaluation of neurovascular relationship by using contrast-enhanced and unenhanced 3D time-of-flight MR angiography in patients with trigeminal neuralgia. Acta Radiol 52(8):894-898

22. Satoh T, Omi M, Nabeshima M, Onoda K, Date I (2009) Severity Analysis of Neurovascular Contact in Patients with Trigeminal Neuralgia: Assessment with the Inner View of the 3D MR Cisternogram and Angiogram Fusion Imaging. American Journal of Neuroradiology 30(3):603-607

23. Maarbjerg S, Wolfram F, Gozalov A, Olesen J, Bendtsen L (2015) Significance of neurovascular contact in classical trigeminal neuralgia. Brain 138(Pt 2):311-319

24. Ramesh VG, Premkumar G (2009) An anatomical study of the neurovascular relationships at the trigeminal root entry zone. J Clin Neurosci 16(7):934-936 


\section{Tables}

Table 1 Patient demographics and clinical characteristics 


\begin{tabular}{|c|c|c|}
\hline Characteristic & $(\mathrm{N}=73)$ & Value \\
\hline Age at surgery, yrs & & $60 \pm 9$ \\
\hline \multicolumn{3}{|l|}{ Gender, n (\%) } \\
\hline Male & \multicolumn{2}{|l|}{$24(32.9 \%)$} \\
\hline Female & \multicolumn{2}{|l|}{$49(61.7 \%)$} \\
\hline \multicolumn{3}{|c|}{ Side of symptom, n (\%) } \\
\hline & Left & $34(46.6 \%)$ \\
\hline & Right & $39(53.4 \%)$ \\
\hline \multicolumn{3}{|c|}{ Distribution of pain, $\mathrm{n}(\%)$} \\
\hline & V1 only & $2(2.7 \%)$ \\
\hline & V2 only & $36(49.3 \%)$ \\
\hline & V3 only & $12(16.4 \%)$ \\
\hline & $\mathrm{V} 1$ and $\mathrm{V} 2$ & $5(6.8 \%)$ \\
\hline & V2 and V3 & $16(21.9 \%)$ \\
\hline & V1-V3 & $1(1.4 \%)$ \\
\hline Preoperative dura & ation of symptom, yrs & $4.8 \pm 4.2$ \\
\hline \multicolumn{3}{|c|}{ Previous medication treatment, n (\%) } \\
\hline & Carbamazepine & $59(80.8 \%)$ \\
\hline & Phenytoin & $3(4.1 \%)$ \\
\hline & Gabapentin & $9(12.3 \%)$ \\
\hline & Oxcarbazepine & $13(17.8 \%)$ \\
\hline & Tramadol & $1(1.4 \%)$ \\
\hline \multicolumn{3}{|c|}{ Previous treatment, $\mathrm{n}(\%)$} \\
\hline & Block & $3(4.1 \%)$ \\
\hline & Radiofrequency ablation & $6(8.2 \%)$ \\
\hline & Endotoxin injection & $2(2.7 \%)$ \\
\hline & Acupuncture & $4(5.5 \%)$ \\
\hline
\end{tabular}


Table 2 Offending vessel distribution

\begin{tabular}{|lll|}
\hline Offending vessel & Symptomatic side & Asymptomatic side \\
\hline Posterior inferior cerebellar artery (PICA) & 4 & 0 \\
\hline Superior cerebellar artery (SCA) & 33 & 19 \\
\hline Anterior inferior cerebellar artery (AICA) & 6 & 0 \\
\hline SCA+PICA & 1 & 0 \\
\hline Vertebral artery (VA) & 2 & 0 \\
\hline PICA+AICA & 1 & 0 \\
\hline SCA+AICA & 2 & 0 \\
\hline Arteriovenous mixed & 16 & 0 \\
\hline SCA+VA & 0 & 3 \\
\hline Vein & 7 & 11 \\
\hline Arachnoid adhesion & 1 & 0 \\
\hline
\end{tabular}

\section{Table 3 Cut-off vale of NVC score}

\begin{tabular}{|lll|}
\hline Cut-off value of NVC score & Sensitivity $(95 \% \mathrm{Cl})$ & Specificity $(95 \% \mathrm{Cl})$ \\
\hline$>2$ & $100.0(95.1-100.0)$ & $60.3(48.1-71.5)$ \\
\hline$>3$ & $87.7(77.9-94.2)$ & $91.8(83.0-96.9)$ \\
\hline$>4^{*}$ & $82.2(71.5-90.2)$ & $98.6(92.6-100.0)$ \\
\hline$>5$ & $67.1(55.1-77.7)$ & $100.0(95.1-100.0)$ \\
\hline AUC=Area under curve; $95 \% \mathrm{Cl}=95 \%$ confidence interval; NVC=Neurovascular compression. \\
* refers to the optimal cut-off value in predicting trifacial neuralgia \\
\hline
\end{tabular}

\section{Figures}




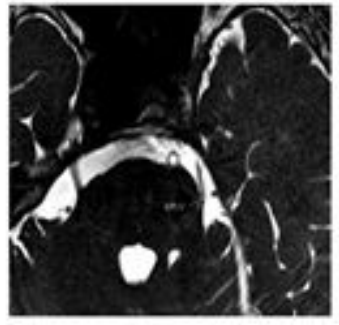

(A)

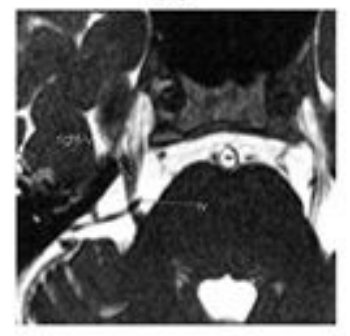

(D)

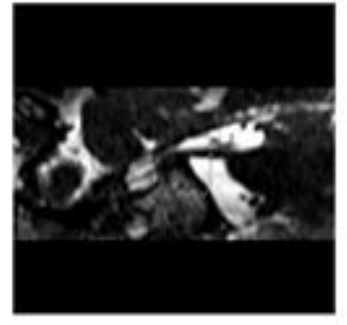

(B)

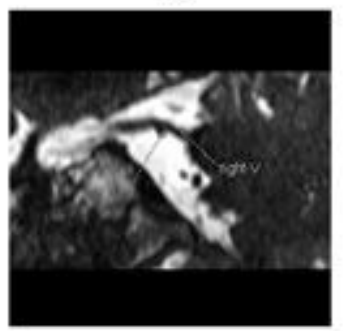

(E)

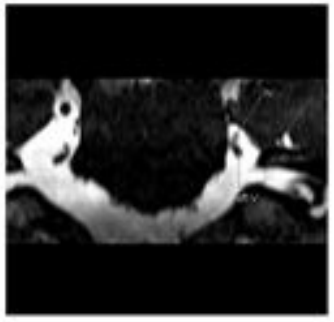

(C)

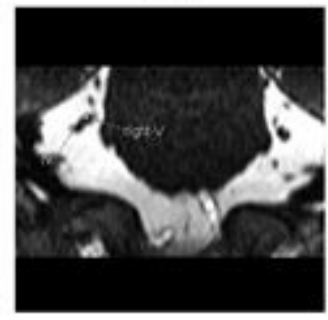

(F)

\section{Figure 1}

Case illustration of NVC. Upper panel: 3D-FIESTA with axial (A), oblique sagittal (B), and coronal (C) images of a 68-year-old female: the NVC score of (A), (B), and (C) were 1, 1 and 1, respectively. The overall NVC score of this patient was 3. Lowe panel: 3D-FIESTA with axial (D, oblique sagittal (E), and coronal (F) images of a 60-year-old female: the NVC score of (A), (B), and (C) were 3, 3 and 3, respectively. The overall NVC score of this patient was 9; yellow arrow: trigeminal nerve; red arrow: offending vessel

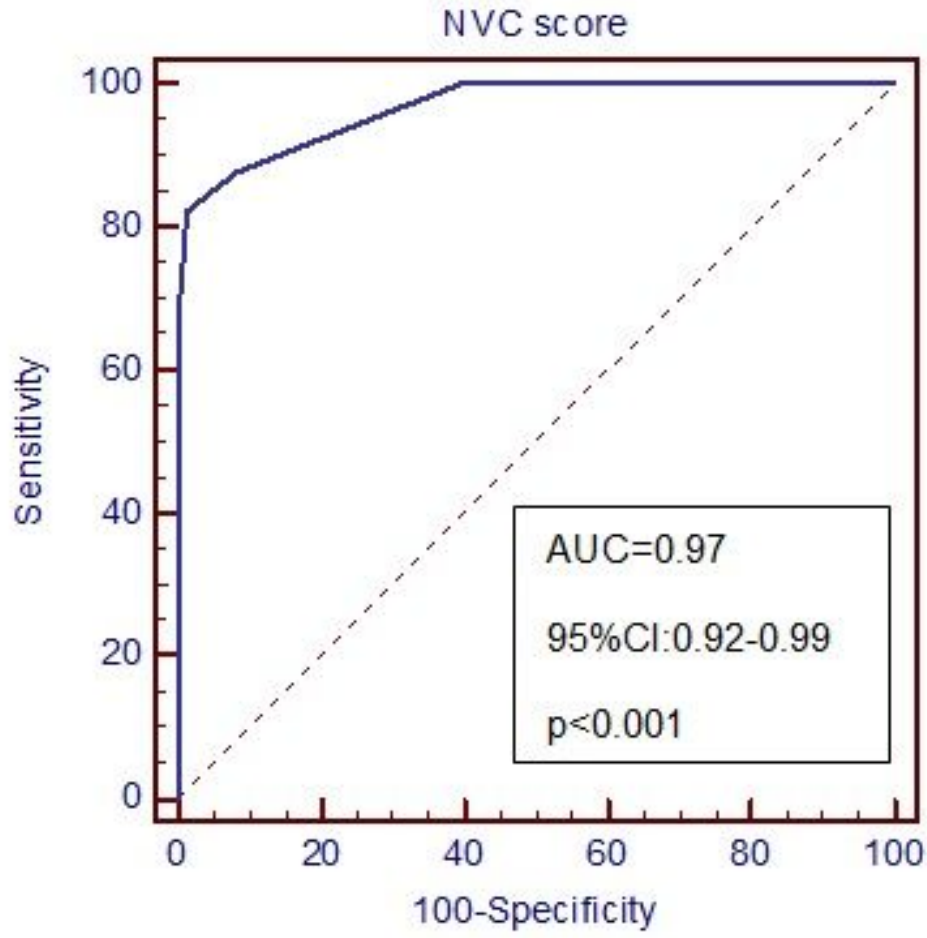

Figure 2 
ROC curve of NVC score in predicting trifacial neuralgia

Page 15/15 